\title{
Type three secretion system effector translocation: one step or two?
}

\section{Charlotte A. Perrett and Daoguo Zhou*}

Department of Biological Sciences, Purdue University, West Lafayette, IN, USA

*Correspondence: zhoud@purdue.edu

\section{A commentary on}

Translocation of surface-localized effectors in type III secretion

by Akopyan, K., Edgren, T., Wang-Edgren, H., Rosqvist, R., Fahlgren, A., Wolf-Watz, H., and Fallman, M. (2011). Proc. Natl. Acad. Sci. U.S.A. 108, 1639-1644.

Type three secretion systems (T3SSs) are macromolecular structures utilized by a number of bacterial pathogens to transfer proteins from the bacterial cytosol, across bacterial and host membranes, into host cells (Cornelis, 2006). The process of crossing the bacterial membranes is termed secretion with the steps of crossing the host membranes being called translocation. Once inside the host cells, the bacterial proteins subvert normal cellular processes to benefit the bacterium; the specific effect dependent on the infectious cycle of the bacterium. For example, Salmonella enterica uses effector proteins to remodel the host cell actin cytoskeleton and plasma membrane, mediating its uptake into nonphagocytic cells (Ly and Casanova, 2007).

Type three secretion systems are highly conserved and consist of approximately 20 proteins that assemble the basal body and needle components of a functional T3SS. The basal body is believed to carry out secretion and the hollow channel inside the needle structure is presumed to act as a conduit through which bacterial proteins enter the host cell (Kubori et al., 1998; Marlovits et al., 2004; Cornelis, 2006). Although various models have been proposed for the secretion apparatus, there is very little evidence detailing the translocation mechanism. The current prevailing model is that the effectors transport from the needle tip across the host cell membrane using bacterial proteins that create a translocon pore in the host cell membrane (Hakansson et al., 1996; Scherer et al., 2000), thus completing secretion and translocation in a "one-step" process. In a recent publication, Akopyan et al. (2011) provide evidence challenging this canonical "one-step" model for the transport of effector proteins, proposing that in Yersinia pseudotuberculosis at least, a "two-step" model of T3SS-dependent protein translocation may be possible. In this "two-step" process, bacterial effectors are first secreted to the surface of the bacterium and then translocated across the host cell membrane in a T3SS-dependent manner.

Immunoelectron microscopy showed that Yersinia T3SS effectors YopE and YopH, and the translocator YopD are evenly distributed across the Yersinia surface prior to contact with host cells (Akopyan et al., 2011). It is not uncommon for translocator proteins to be found on the bacterial surface (Ménard et al., 1994; Watarai et al., 1996; Lara-Tejero and Galan, 2009), particularly since the hydrophilic translocator protein forms a tip at the end of the T3SS needle (Mueller et al., 2008). However, previous data employing immunofluorescent microscopy indicated that effector proteins accumulated in the bacterial cytoplasm awaiting exit through the T3SS, and often exhibited polarized localization toward active T3SS needles (Schlumberger et al., 2005; Jaumouille et al., 2008; Winnen et al., 2008). Such data fits with a one-step T3SS translocation model where T3SS substrates are synthesized and stored in the bacterial cytoplasm ready to be fed to the T3SS basal body upon T3SS activation, with subsequent transport through the interior of the needle. While there is no direct evidence for effector transport through the needle, experiments have shown the need for effectors to unfold prior to secretion indicative of transport though the needle core (Akeda and Galan, 2005). A one-step model would also explain how effectors can be translocated in a hierarchial manner as has been shown for SipA, SopE, and SptP in Salmonella (Winnen et al., 2008; Lara-Tejero et al., 2011). Perhaps the most compelling evidence to support the current "one-step" model is that most T3SS pathogens cannot be cross-complemented, that is, a mixed infection with mutants deficient in secretion and translocation separately will not restore the wild-type phenotype. Thus, the finding that functional effectors are present on the Yersinia surface and can be translocated to induce physiological responses in host cells is surprising and requires reappraisal of the current data.

Akopyan et al. (2011) presented evidence that coating a $Y$. pseudotuberculosis yopH mutant with YopH in vitro restores the mutant to inducing a similar physiological response to wild-type bacteria. Furthermore, use of YopH-Bla or YopK-Bla hybrid proteins to coat various Yersinia mutants led to the rapid, specific T3SS-specific translocation of the surface effectors into host cells. It is intriguing that surface effectors appear to be translocated in a manner similar to secreted effectors. It is still unclear how these surface effectors are transferred to the host cell membrane but it is clear that the translocon remains important for their transfer inside the host cell; a Yersinia mutant lacking YopB and YopD failed to promote translocation of a YopH-Bla coat, and the surface effectors require a translocation domain for transport inside the host cell (Akopyan et al., 2011). This led the authors to propose that the translocon recognizes surface effectors via this domain and can then feed them into the host cell. This also explains why effectors possess such a domain. Hitherto, it was unclear why the translocon domain was required in addition to a secretion signal if the translocon acted as a continuation of the T3SS needle. This does not rule out the potential for the translocon to act as a checkpoint in T3SS secretion and ensure the smooth transition of effectors across the host cell membrane in both a one-step and two-step model. Indeed, relatively little is known as to how exactly the translocon functions, which could shed further light on the necessity of translocation domains in effector proteins. The additional finding that 
the YopH secretion signal is not required for translocation supports the ability for T3SS substrates to be located on the bacterial surface prior to their translocation in a T3SS manner (Akopyan et al., 2011). The possession of both secretion and translocation sequences by T3SS effectors may indicate a flexibility in T3S hitherto unknown, and may suggest one-step and two-step translocation occur at the same time, although it is not clear yet what the advantage may be during infection.

One of the major questions this latest research poses is how universal two-step translocation is to other bacteria possessing T3SSs. Initial experiments coating Salmonella Typhimurium with YopH-Bla constructs showed Salmonella was able to translocate YopH, in a SPI-1 T3SSdependent manner (Akopyan et al., 2011). A more definitive experiment would be to demonstrate whether such translocation could restore a biological phenotype, such as ruffling conferred by SopB or SopE during Salmonella infection. Given the homology that exists between components and effectors of the Yersinia, Salmonella, and Shigella T3SSs (Rosqvist et al., 1995) it would not be surprising if the two-step translocation mechanism was used by each. Indeed, the Salmonella effector SptP has homology with both YopE and YopH (Kaniga et al., 1996) which would make it a likely candidate for two-step translocation. However, homology alone will not dictate that this translocation method is universal. The interactions of Yersinia, Salmonella, and Shigella with host cells leads to different outcomes, reflecting the different lifestyles led by these bacteria that have ultimately shaped their effector repertoire and may therefore potentially have individualized the function of their T3SSs. It will be necessary now to determine whether two-step translocation is universal to some or all T3SS-possessing bacteria in addition to elucidating the role of this process in infection. Hints may come from determining what proportion of effectors are targeted to the bacterial surface, whether all effectors are represented on the surface and of course how these proteins are transported to the surface. With the advancement in imaging technology, it is not impossible to speculate that we might be able to visualize both the secretion and the translocation process in the near future and to be able to demonstrate the "one-step" and/or "two-step" translocation beyond reasonable doubt.

\section{REFERENCES}

Akeda, Y., and Galan, J. E. (2005). Chaperone release and unfolding of substrates in type III secretion. Nature 437, 911-915.

Akopyan, K., Edgren, T., Wang-Edgren, H., Rosqvist, R., Fahlgren, A., Wolf-Watz, H., and Fallman, M. (2011). Translocation of surface-localized effectors in type III secretion. Proc. Natl. Acad. Sci. U.S.A. 108, 1639-1644.

Cornelis, G. R. (2006). The type III secretion injectisome. Nat. Rev. Microbiol. 4, 811-825.

Hakansson, S., Schesser, K., Persson, C., Galyov, E. E., Rosqvist, R., Homble, F., and Wolf-Watz,H. (1996). The YopB protein of Yersinia pseudotuberculosisis essential for the translocation of Yop effector proteins across the target cell plasma membrane and displays a contact-dependent membrane disrupting activity. EMBO J. 15, 5812-5823.

Jaumouille, V., Francetic, O., Sansonetti, P. J., and Tran Van Nhieu, G. (2008). Cytoplasmic targeting of IpaC to the bacterial pole directs polar type III secretion in Shigella. EMBO J. 27, 447-457.

Kaniga, K., Uralil, J., Bliska, J. B., and Galán, J. E. (1996). A secreted protein tyrosine phosphatase with modular effector domains in the bacterial pathogen Salmonella typhimurium. Mol. Microbiol. 21, 633-641.

Kubori, T., Matsushima, Y., Nakamura, D., Uralil, J., LaraTejero, M., Sukhan, A., Galán, J. E., and Aizawa, S. I. (1998). Supramolecular structure of the Salmonella typhimurium type III protein secretion system. Science 280, 602-605.

Lara-Tejero, M., and Galan, J. E. (2009). Salmonella enterica serovar typhimurium pathogenicity island 1-encoded type III secretion system translocases mediate intimate attachment to nonphagocytic cells. Infect. Immun. 77, 2635-2642.

Lara-Tejero, M., Kato, J., Wagner, S., Liu, X., and Galan, J. E. (2011). A sorting platform determines the order of protein secretion in bacterial type III systems. Science 331, 1188-1191.

Ly, K. T., and Casanova, J. E. (2007). Mechanisms of Salmonella entry into host cells. Cell. Microbiol. 9, 2103-2111.

Marlovits, T. C., Kubori, T., Sukhan, A., Thomas, D. R., Galán, J. E., and Unger, V. M. (2004). Structural insights into the assembly of the type III secretion needle complex. Science 306, 1040-1042.

Ménard, R., Sansonetti, P., Parsot, C., and Vasselon, T. (1994). Extracellular association and cytoplasmic partitioning of the IpaB and IpaC invasins of S. flexneri. Cell 79, 515-525.

Mueller, C. A., Broz, P., and Cornelis, G. R. (2008). The type III secretion system tip complex and translocon. Mol. Microbiol. 68, 1085-1095.

Rosqvist, R., Persson, C., Hakansson, S., Nordfeldt, R., and Wolf-Watz, H. (1995). Translocation of the Yersinia YopE and YopH virulence proteins into target cells is mediated by YopB and YopD. Contrib. Microbiol. Immunol. 13, 230-234.

Scherer, C. A., Cooper, E., and Miller, S. I. (2000). The Salmonella type III secretion translocon protein SspC is inserted into the epithelial cell plasma membrane upon infection. Mol. Microbiol. 37, 1133-1145.

Schlumberger, M. C., Muller, A. J., Ehrbar, K., Winnen, B., Duss, I., Stecher, B., and Hardt, W.-D. (2005). Realtime imaging of type III secretion: Salmonella SipA injection into host cells. Proc. Natl. Acad. Sci. U.S.A. 102, 12548-12553.

Watarai, M., Funato, S., and Sasakawa, C. (1996). Interaction of Ipa proteins of Shigella flexneri with alpha5beta1 integrin promotes entry of the bacteria into mammalian cells. J. Exp. Med. 183, 991-999.

Winnen, B., Schlumberger, M. C., Sturm, A., Schupbach, K., Siebenmann, S., Jenny, P., and Hardt, W. D. (2008). Hierarchical effector protein transport by the Salmonella Typhimurium SPI-1 type III secretion system. PLoS ONE 3, e2178. doi: 10.1371/journal.pone.0002178

Received: 28 February 2011; accepted: 04 March 2011; published online: 16 March 2011.

Citation: Perrett CA and Zhou D (2011) Type three secretion system effector translocation: one step or two? Front. Microbio. 2:50. doi: 10.3389/fmicb.2011.00050

This article was submitted to Frontiers in Cellular and Infection Microbiology, a specialty of Frontiers in Microbiology.

Copyright (c) 2011 Perrett and Zhou. This is an openaccess article subject to an exclusive license agreement between the authors and Frontiers Media SA, which permits unrestricted use, distribution, and reproduction in any medium, provided the original authors and source are credited. 